\title{
Interacciones entre proposiciones condicionales y sistemas matemáticos de símbolos en una tarea matemática
}

\author{
Interactions between conditional propositions and mathematical \\ symbol systems in a mathematical task
}

\section{Eduardo Mario Lacues Apud, ${ }^{1}$ Leonora Díaz Moreno, ${ }^{2}$ Juan Antonio Huertas ${ }^{3}$}

\begin{abstract}
Resumen: Se presentan resultados de una investigación con estudiantes de un curso de Cálculo de primer año universitario, diseñada para indagar acerca del uso que ellos hacen de los enunciados condicionales (condición suficiente, condición necesaria) y su relación con los Sistemas Matemáticos de Símbolos en los que se presenta la información sobre estos enunciados (registro gráfico, registro algebraico). Las preguntas formuladas en esta instancia son similares a las que se deben responder en actividades relacionadas con la noción de función. Se encontraron diferencias en el rendimiento de los estudiantes, que indican que las tareas sobre condición necesaria presentadas en el registro gráfico resultan significativamente más sencillas que las demás; hay indicios de un mejor rendimiento en tareas presentadas en el registro gráfico respecto de las correspondientes al algebraico y en las tareas sobre condición necesaria en relación con las respectivas sobre condición suficiente.
\end{abstract}

Fecha de recepción: 06 de mayo de 2019. Fecha de aceptación: 10 de enero de 2021

1 Departamento de Ciencias Exactas y Naturales, Universidad Católica del Uruguay (UCU), elacues@ gmail.com, orcid.org/0000-0001-7449-999X

2 Pontificia Universidad Católica de Chile, leonoradiazmoreno@gmail.com, orcid.org/0000-0001-5765-6332

3 Departamento de Psicología Básica, Universidad Autónoma de Madrid (UAM), Juanantonio.huertas@ uam.es, orcid.org/0000-0002-1518-8398 
Palabras clave: Condición necesaria, Condición suficiente, Sistemas Matemáticos de Símbolos, Registros de Representación, Funciones.

\begin{abstract}
Results from an investigation with freshmen in a first year university Calculus course are presented, which was designed to inquiry about their use of conditional propositions (necessary condition, sufficient condition) and its relation with the Mathematical Symbol System used to present information about these propositions (graphic register, algebraic register). The questions formulated in this instance are similar to those that must be answered in activities related with the notion of function. Differences in students' performance indicate that tasks about necessary condition on graphic register result significantly easier than others; there is evidence of a better performance in tasks on graphic register in relation with algebraic one, and in tasks about necessary condition with respect to sufficient condition.
\end{abstract}

Keywords: Necessary condition, Sufficient condition, Mathematical Symbol Systems, Representations registers, Functions.

\title{
INTRODUCCIÓN
}

Estudios desde principios de los noventa a la fecha reportan de modo persistente, problemas de los ingresantes a la universidad con el aprendizaje de las Matemáticas (Tall, 1990; Artigue, 2000; Camarena, 2010) y, en particular, dificultades con conceptos formalizados para los que son insuficientes sus recursos previos. Por ejemplo, para construir un pensamiento analítico que considere sus habilidades algebraicas previas, deben abordar las brechas de igualdad y de razonamiento (Artigue, 1995): se requiere añadir, a una igualdad resultado de equivalencias algebraicas, una igualdad resultado de proximidad, y a un razonar por equivalencias sucesivas, un razonamiento por condiciones suficientes.

Por su parte, la actividad con la noción de condicional y, específicamente, la distinción entre condición necesaria y condición suficiente, es crucial para los estudiantes ingresantes a programas científico-tecnológicos, dada su presencia en argumentaciones y demostraciones en los cursos iniciales de Matemáticas.

Algunos informes de investigación revelan dificultades que enfrentan estos estudiantes. Los novatos tienen formas de aproximación a la noción de 
demostración basadas en consideraciones contextuales o intuitivas (Camacho, Sánchez y Zubieta, 2014) donde la relevancia de la estructura lógica no es reconocida, a diferencia de lo que hacen los expertos que se preocupan por reconocer la validez de las inferencias realizadas (Inglis y Alcock, 2012).

Lew, Fukawa-Conelly, Mejía-Ramos y Weber (2016) señalan que la mirada de un profesor sobre una demostración, centrada en ideas cruciales que la organizan, suele no coincidir con la de sus estudiantes focalizada más bien en los detalles de los cálculos que se realizan; indican que las acepciones técnicas de ciertos términos no son compartidas por profesor y estudiantes. La conjunción de estos factores puede explicar por qué los estudiantes podrían no comprender lo que la lección de su profesor está enseñando.

En otro orden, algunos estudios han proporcionado información acerca de estrategias usadas por estudiantes en los procesos de construir o escribir demostraciones.

Weber y Alcock (2004) han distinguido entre pruebas sintácticas y pruebas semánticas; en las primeras, el sujeto manipula formalmente las representaciones simbólicas hasta obtener la conclusión mientras que, en las segundas el ejecutante apela a instanciaciones ${ }^{4}$ de los objetos matemáticos a los que la prueba refiere para avanzar hacia la solución.

Zazkis, Weber y Mejía-Ramos (2015) identificaron dos estrategias de abordaje usadas por estudiantes de Matemáticas al construir pruebas y las relacionaron con los procesos de resolución de problemas:

Cuando usan una estrategia orientada a un objetivo los estudiantes estarían desarrollando una comprensión profunda de la proposición que estén probando, eligiendo un plan con base en esa comprensión, desarrollando un argumento gráfico acerca de la validez de la proposición y formalizando este argumento en una prueba. Cuando usen una estrategia superficial los estudiantes estarían comenzando a probar diferentes planes inmediatamente luego de leer la proposición y abandonarían cada uno de los planes a la primera señal de dificultad. ${ }^{5}$ (p. 12)

Es interesante destacar que en la primera estrategia descrita, los estudiantes construyen argumentos gráficos que luego formalizan. Esto puede interpretarse

4 "Por instanciación nos referimos a una manera sistemáticamente repetible por la que el individuo piensa en un objeto matemático, que es internamente significativa para ese individuo." Traducción de los autores.

${ }^{5}$ Traducción de los autores 
en términos de los procesos de conversión entre registros semióticos de representación (Duval, 1998), que se discutirán más adelante. La construcción de diagramas o gráficas también es mencionada por Weber y Alcock (2004) como una de las posibles formas de instanciación.

Estos antecedentes conducen a distinguir dos elementos que interactúan en los procesos de construcción o en la aceptación de la validez de una demostración. Por un lado, las reglas de inferencia que permiten justificar los pasos sucesivos de una prueba a partir de los anteriores dependen de la definición del valor de verdad del condicional, cuyas características técnicas son frecuentemente causa de dificultades. Por otro, las formas de representación que los ejecutantes asumen para llevar adelante la tarea parecen tener incidencia en el éxito conseguido.

Responder a la interrogante de la influencia del registro de representación, sea algebraico o gráfico, en la dificultad de la tarea con el condicional, aportaría antecedentes para diseños de enseñanza que podrían contribuir a mejorar los desempeños de estudiantes ingresantes.

En este trabajo se presenta una investigación desarrollada en un curso de Cálculo en primer semestre de carreras de ingeniería, destinada a estudiar dos de los aspectos que se han destacado y se señalan a continuación.

En primer término, interesó conocer qué uso de la noción de condicional hacen los alumnos ingresantes a la universidad, en particular, de la distinción entre condición necesaria y condición suficiente. En segundo lugar, se propuso responder a la interrogante acerca de la influencia que pudiera tener el registro de representación en el que se muestra la consigna (algebraico o gráfico) en la dificultad de la tarea. Desde el punto de vista didáctico estas dos cuestiones son relevantes. Tienen implicaciones sobre la elección de estrategias de enseñanza y la forma de presentación de tareas a los estudiantes, entre otros aspectos.

Los trabajos fueron planteados sobre los contenidos matemáticos de imagen y preimagen por medio de una función. Se eligió la noción de función porque permite fácilmente presentar tareas en los registros algebraico y gráfico. Asociadas a esta noción, las de imagen y preimagen brindan oportunidades para formular consignas en las que el reconocimiento de condiciones necesarias o suficientes es crucial, así como las instancias de cuantificación que en ellas aparecen.

En la sección siguiente se introducen nociones sobre los Sistemas Matemáticos de Símbolos (SMS). A continuación de ésta, se discute con cierto detalle cuestiones relativas a la argumentación matemática con base en el condicional. Luego de estas dos secciones se informa acerca del diseño metodológico, el procesamiento de los datos y las conclusiones extraídas. 


\section{SISTEMAS MATEMÁTICOS DE SÍMBOLOS Y REGISTROS DE REPRESENTACIÓN}

Al hablar de Sistemas Matemáticos de Símbolos, la ubicación del adjetivo "Matemáticos" calificando al sustantivo "Sistemas" es deliberada y pretende poner énfasis en que no son los símbolos los que se consideran matemáticos, sino los sistemas que integran ciertos símbolos y una lengua vernácula. Esta es la posición que defiende Puig (2003) al señalar que quien dota de significado al texto es el sistema y no los signos ni la lengua por sí mismos. Enfatiza de este modo la necesidad de considerar no los signos de manera individual, sino la entidad constituida por un conjunto de signos (no necesariamente lingüísticos) junto con una cierta lengua vernácula, cuyas configuraciones cobran sentido en un proceso social por el que se van produciendo consensos en la actividad matemática.

Tomando como referencia a Palmer (1977) la definición de SMS fue establecida por Kaput (1987) como un sistema de representación. En esta definición es central la noción de esquema simbólico, caracterizado como el par formado por una colección de caracteres y otra de reglas que establecen la forma en que pueden combinarse, ordenarse o transformarse estos caracteres. La colección de reglas conforma la sintaxis del esquema simbólico.

Siguiendo esta formulación, un sistema de símbolos es una terna formada por esquema simbólico, un campo de referencia y una correspondencia entre ellos, que podría no ser biunívoca. Cuando el campo de referencia es una estructura matemática, se tiene un Sistema Matemático de Símbolos.

Al comentar este desarrollo, Radford (1998) Ilama la atención sobre una dualidad que Kaput atribuye a los SMS: por un lado, éstos median en las actividades de comunicación gracias a su condición de ser compartidos por la comunidad; por otro, intervienen en el funcionamiento cognitivo individual de una forma que Kaput (1987) mismo refiere así: "[...] con las acciones más tempranas y las estructuras cristalizadas en símbolos estables y concretamente manipulables, la mente es liberada para actuar sobre o reflexionar acerca de esas acciones y estructuras en formas nuevas, tal vez conduciendo a otro círculo de construcciones matemáticas". ${ }^{6}$ (p. 162)

Es decir, en este proceso, los SMS se convierten en portadores de significados que resumen conjuntos (eventualmente ordenados) de acciones sobre objetos matemáticos, y pasan a ser a su vez posibles participantes en nuevas construcciones matemáticas, susceptibles de representación. Por su parte, en la

\footnotetext{
${ }^{6}$ Traducción de los autores
} 
caracterización de Martí y Pozo (2000) los SMS resultan ser una clase de sistemas externos de representación: tienen existencia independiente de su creador, permanecen en el tiempo a la vez que se organizan espacialmente, y son el resultado de consensos. El problema de la representación ha sido estudiado desde diferentes perspectivas en el ámbito de la enseñanza y el aprendizaje de la Matemática: vinculando la función comunicacional de los SMS con la cognitiva (Radford, 1998), lo que se complementa con el señalamiento que hace Berger (2004) en el sentido de que el uso comunicacional de los signos evoluciona hacia una coincidencia entre los significados personales de los conceptos matemáticos y los sociales sostenidos por la comunidad matemática; Arcavi (2003) enfatiza que diferentes representaciones aportan visiones complementarias, lo que se asocia con el éxito en resolución de problemas mediante la habilidad para articular diferentes representaciones (Gagatsis y Shiakalli, 2004) y con el aprendizaje a través de la generación de representaciones propias y consensuadas con los pares (Selling, 2015), cuestión que también ha sido estudiada en otras disciplinas (Pocoví y Collivadino, 2014). Los desempeños de los estudiantes al usar en forma casi exclusiva un sistema de representación (algebraico o gráfico) han sido también reportados (Alcock y Simpson, 2004; 2005) mientras que la emergencia de objetos matemáticos y las dificultades asociadas con los procesos de conversión entre registros han sido también estudiados (Rojas, 2015). Estos puntos de vista ayudan a situar el problema de la apropiación por parte de los estudiantes (en nuestro caso, universitarios) de los objetos matemáticos con los que toman contacto a través de los signos (palabras, símbolos matemáticos) que integran el discurso matemático. Este discurso está conformado en torno a un conjunto de consensos, que dota de un significado preciso, compartido por la comunidad matemática, a las expresiones que se usan para comunicar resultados o argumentar en torno a la validez de enunciados en Matemática.

Dado que los problemas que han dado origen a estas formas de representación suelen ser ajenos a los estudiantes, es difícil que las estructuras comunicativas que finalmente han sido consensuadas sean visibles para ellos y pueden resultarles artificiales. En la clase junto con este discurso coexisten otros, asociados con la intención de enseñar y de aprender, que no necesariamente son concurrentes, sino que en ocasiones presentan divergencias tan marcadas que suponen un obstáculo en la aprehensión de los saberes por parte de los aprendices. Si bien la preocupación que genera esta situación es de larga data (Kieran, 1992) la dificultad que implica para cada principiante apropiarse de este 
discurso para usarlo de manera competente es de las menos atendidas desde la enseñanza de las Matemáticas. Por otro lado, la consideración del desarrollo histórico del uso de símbolos en Matemática permite ampliar la perspectiva. A partir de un trabajo de Vieta sobre una traducción al latín de los documentos de Diofanto, sitúa Kieran (1992) a fines del siglo XV el comienzo de la etapa simbólica, la última de las tres en la que separa la historia de la evolución del simbolismo algebraico. Según esta autora, Diofanto marca el final de la primera, que ella llama retórica y está caracterizada por una ausencia total de formalismo, y el comienzo de la segunda. Ésta se extiende hasta el siglo XV, cuando en Europa a partir de los procedimientos diofánticos comienzan a elaborarse métodos generales para abordar la resolución de diferentes problemas algebraicos.

En apoyo a esta descripción, Radford y Puig (2007) señalan que en sus orígenes en Mesopotamia, el álgebra trataba las cantidades desconocidas (las variables en un sentido actual) como elementos contextualizados, lo que permitía asignarles naturalmente un significado (por ejemplo, geométrico) y otorgaba medios para comprobar la validez de los cálculos efectuados (siguiendo con el ejemplo, mediante la transformación del problema geométrico asociado). Esta asociación de lo algebraico con lo geométrico se mantuvo hasta el Renacimiento. La solución de un problema, en esta etapa retórica, está fuertemente atada al contexto y resulta, por lo tanto, particular. Por eso, no se perciben todavía procesos de abstracción o generalización que permitan reconocer estructuras matemáticas, algoritmos o estrategias de resolución. La formulación verbal se complica gradualmente al extremo que podría ser muy confuso expresar los procesos que se siguen. En una etapa como la de Diofanto, la representación de los procesos se ha hecho a través de símbolos, pero aún no se expresan éstos en toda su generalidad. Por eso, la relación del ejecutante con el proceso de solución del problema, si bien no es tan directa como en el caso retórico, conserva cierta cercanía, manifestada en el mantenimiento de los datos numéricos, lo que transforma al proceso en una solución para ese caso, aun cuando pueda vislumbrarse lo que sería una generalización.

En la etapa simbólica, la distancia entre el ejecutante y la tarea se ha agrandado, y los procesos que se siguen pueden plantearse en términos abstractos: ya no se opera con números, sino con variables. Llegados a este punto, el propio procedimiento puede ser objeto de generalización, de manera que se extienda a otras situaciones. Un aporte para avanzar en el desarrollo de la lógica aristotélica fue el trabajo de George Boole para intentar algebrizar el cálculo de predicados. Como señala Glymour (1998) uno de los postulados asumidos por 
Boole era que las leyes del pensamiento tenían una forma algebraica. El trabajo de Boole significó el primer desarrollo importante en Lógica desde Aristóteles. Sin embargo, pese a su magnitud, no pudo resolver ciertas cuestiones relacionadas con la noción de prueba, en particular aquéllas donde aparecen variables individuales cuantificadas, como las relacionadas con el límite matemático. El resultado de tales procesos históricos, en opinión de Radford y Puig (2007) a través de lo que denominan "Embedment Principle", es un sistema algebraico constituido por un conjunto de reglas sintácticas y significados portadoras del funcionamiento cognitivo de generaciones anteriores:

Esta actividad cognitiva histórica depositada en signos, el sistema semiótico que ellas forman, y las prácticas sociales que ellas median ofrecen a nuestros estudiantes ciertas líneas de desarrollo conceptual, vectores maleables de crecimiento cognitivo que los estudiantes pueden seguir y transformar de acuerdo con las actividades en las que se involucren. ${ }^{7}$ (Radford y Puig, p. 148)

Esto es, la actividad histórico-cognitiva asentada en signos, el sistema semiótico que forman y las prácticas sociales que median, ofrecen a los estudiantes direcciones de desarrollo y formas de apropiación del saber escolar del Álgebra. El logro de dominio en el uso de SMS por los aprendices, permite tomar distancia de las formas más concretas de formulación de los problemas. Llevado a un extremo, esto puede significar que los estudiantes sean capaces de ejecutar algoritmos correctamente, sin tener en cuenta los problemas a los que esos algoritmos originalmente dieron respuesta. Esto comporta el riesgo de confundir la habilidad en la ejecución de rutinas de cálculo con la comprensión conceptual del problema, que incluye, entre otros elementos, la interpretación de los significados de los símbolos utilizados y de las relaciones que con su uso se establecen entre las entidades involucradas. Vale la pena destacar que otra de las características que los SMS comparten con el lenguaje, es que muchos de sus significados dependen del contexto en el que se formulan las sentencias. En cada caso, las propiedades que se resumen en esos SMS son diferentes, y para cada aprendiz constituye un problema identificar, a partir del contexto en el que están siendo usadas, cuáles son las pertinentes. Regine Douady (1984) introdujo en su tesis doctoral la noción de "juego de cuadros" para aludir a contextos de formulación de sentencias matemáticas y destacó la

7 Traducción de los autores. 
importancia de promover desde la enseñanza las competencias de los aprendices para transitar entre estos "cuadros" o "marcos". Ella justifica este énfasis en los procesos de cambio de cuadro a partir de dos principios: el de que todo concepto matemático es susceptible de ser representado en más de un marco, y el de que estas representaciones no coinciden, dado que cada representación favorece la comprensión de algún aspecto del concepto que resulta menos claramente aprehensible en otra representación.

Duoady avanzó en lo que quería decir al referirse a un marco (Douady, 1986), estableciendo que se conforma con objetos matemáticos, considerando las relaciones que existen entre ellos y las formulaciones que representan a unos y otras, y se manejó con ideas intuitivas a este respecto (marco físico, marco geométrico, marco algebraico, marco numérico, entre otros). Su trabajo fue pionero y disparador de otros desarrollos. Raymond Duval (Duval, 1995; 1998) al introducir la teoría de los registros de representación semiótica, avanzó en el desarrollo de estas nociones. Cada registro semiótico es un sistema de representación en el que es posible ejecutar al menos tres operaciones: el reconocimiento de formulaciones simbólicas como representantes de ciertas entidades, la transformación de una formulación en otra dentro del mismo sistema (tratamiento) y, la transformación de una formulación en un cierto sistema en otra de otro sistema (conversión). En cada registro de representación se configura un SMS que no sólo incorpora los signos propios del registro, sino las reglas de tratamiento y las referencias que permiten los procesos de traducción entre registros. Las operaciones de conversión dan cuenta de los procesos de traducción que permiten el "juego de marcos" o desplazamientos entre registros, es decir, la posibilidad de establecer similitudes, tal vez parciales, entre las representaciones en diferentes registros del mismo concepto matemático. Es pertinente aclarar que, si bien aparece "registro algebraico" como designación de uno de los marcos, podría hablarse más precisamente de "registro analítico-algebraico", para dar cuenta de que las representaciones algebraicas de funciones juegan, además, un papel importante en las nociones variacionales que son propias del estudio del Cálculo.

\section{CONDICIONES NECESARIA Y SUFICIENTE EN LA ARGUMENTACIÓN MATEMÁTICA}

Al problema de decidir si una función es sobreyectiva debe responderse argumentando si dado un elemento del codominio de la función, existe un 
elemento del dominio cuya imagen sea el elemento elegido. En esta situación hay al menos tres señalamientos a hacer.

El primero, es la presencia de cuantificadores, uno universal "[...] dado un elemento [...]", otro existencial. La aparición de éste es evidente, pero la del primero es implícita. El segundo punto destacable es la necesidad de articular el registro de representación en el que se está trabajando con las acciones que se requiere ejecutar para dar la respuesta. El tercer elemento por señalar, es la estructura lógica que está presente en la sentencia a analizar. En nuestro ejemplo, si la función viene dada por medio de una ecuación algebraica, se debe discutir la existencia de soluciones de una cierta ecuación dependiente de un parámetro. En cambio, si la función se representa por medio de una gráfica cartesiana donde se ubicó la variable independiente en el eje de abscisas, la cuestión es decidir si una recta paralela a este eje interseca a la gráfica cuando la ordenada de la recta recorre todos los valores del codominio. El conectivo que aparece es una conjunción: se interroga acerca de la existencia de un elemento que esté en el dominio y cuya imagen sea el elemento dado en el codominio. Como en el caso de los cuantificadores, este conectivo puede aparecer de manera poco explícita. Otra situación similar se da en la actividad para probar que una función tiene límite en un punto. En el currículo de los primeros cursos de Cálculo en carreras en ingeniería en Uruguay, esta definición ocupa un lugar importante: se usa para probar que ciertas funciones tienen un cierto límite en un punto, o para demostrar reglas que proporcionan procedimientos para el cálculo de límites, o para justificar resultados de interés intra-matemático, como la unicidad del límite en caso de existir. En el proceso de usar la definición para justificar la existencia de un cierto límite, es necesario y suficiente verificar que, si se quiere conseguir que la variable dependiente esté en cierto conjunto arbitrariamente elegido, alcanza con encontrar un conjunto adecuado en el que esté la variable independiente. Como antes, hay varias apariciones de cuantificadores y una estructura lógica basada en el conectivo condicional. En efecto, la definición de límite de una función en un punto, tal como quedó establecida a partir de Cauchy, puede escribirse de la siguiente manera:

$\lim _{x \rightarrow a}(x)=b$ si y solo si dado $\varepsilon>0$ existe $\delta>0$ tal que si $0<|x-a|<\delta$ entonces $|f(x)-b|<\varepsilon$. $\mathrm{x} \rightarrow \mathrm{a}$

Explicitando en detalle la traducción de los elementos simbólicos que aparecen en ella, puede redactarse diciendo: 
La función $\mathrm{f}$ tiene límite $\mathrm{b}$ en el punto a si y solo si dado $\varepsilon$ positivo existe $\delta$ positivo de forma que, para cualquier $\mathrm{x}$, si ocurre que la distancia de $\mathrm{x}$ al punto a es positiva y menor que $\delta$ resulta que la distancia de la imagen de $\mathrm{x}$ por medio de $\mathrm{f}$ al número $\mathrm{b}$ es menor que $\varepsilon$.

Por lo tanto, afirmar que una función tiene límite en un punto es equivalente a decir que:

Existe un número b para el cual, dado $\varepsilon$ positivo existe $\delta$ positivo de forma que para cualquier $\mathrm{x}$, si ocurre que la distancia de $\mathrm{x}$ al punto a es positiva y menor que $\delta$ resulta que la distancia de la imagen de $\mathrm{x}$ por medio de $\mathrm{f}$ al número $\mathrm{b}$ es menor que $\varepsilon$.

En definitiva, pueden reconocerse tres instancias de cuantificación en esta definición, dos de ellas más bien explícitas ("dado $\varepsilon>0$ ", "existe $\delta>0$ ") y otra prácticamente oculta ("existe un número b"). Por otro lado, la sentencia cuyo valor de verdad debe ser analizado para decidir acerca de la existencia del límite es un condicional. Ha sido señalado (Durand-Guerrier, 2005; Morou y Kalospyros, s.f.) que este "ocultamiento" de los cuantificadores está asociado con dificultades en la comprensión de los contenidos matemáticos y puede, por eso, inducir a errores en el desempeño de los estudiantes, en particular, en los procesos de demostración y en el uso de las sentencias condicionales, lo que en muchos países ocurre en el momento del ingreso a carreras universitarias en el área de ciencias o tecnologías.

Por su parte, un resultado central en matemáticas es el que establece que el conjunto vacío, representado con la letra nórdica $\emptyset$, está contenido en cualquier otro conjunto. Cuando se examina esta proposición desde la perspectiva de la definición de inclusión de conjuntos, queda claro que un condicional debe ser cierto cuando su antecedente es falso, sin que importe el valor del consecuente. En efecto, decimos que el conjunto X está contenido en el conjunto $\mathrm{Y}$ si y solo si para cualquier $\mathrm{a}$, si a es un elemento de $\mathrm{X}$, entonces es un elemento de $\mathrm{Y}$. En términos formales, $\mathrm{X} \subset \mathrm{Y} \Leftrightarrow(\forall \mathrm{a})(\mathrm{a} \in \mathrm{X} \Rightarrow \mathrm{a} \in \mathrm{Y})$. Concretada al caso en que $\mathrm{X}$ es el conjunto vacío $\emptyset$, la expresión anterior queda: $\emptyset \subset \mathrm{Y} \Leftrightarrow$ $(\forall \mathrm{a})(\mathrm{a} \in \emptyset \Rightarrow \mathrm{a} \in \mathrm{Y})$. Para que la proposición $(\forall \mathrm{a})(\mathrm{a} \in \emptyset \Rightarrow \mathrm{a} \in \mathrm{Y}$ sea cierta, debe ocurrir que $\mathrm{a} \in \emptyset \Rightarrow \mathrm{a} \in \mathrm{Y}$ sea cierta para cualquier a. De acuerdo con la definición de valor de verdad del condicional, esto ocurrirá a menos que $a \in \emptyset$ sea cierto y $\mathrm{a} \in \mathrm{Y}$ sea falso. Ahora bien, por definición, $\mathrm{a} \in \emptyset$ toma el valor falso para cualquier $\mathrm{a}$, en tanto $\mathrm{a} \in \mathrm{Y}$ puede ser tanto falso como cierto, 
dependiendo de a y de Y. De aquí se concluye que $\emptyset$ es un subconjunto de cada conjunto. Esta característica técnica de la definición del valor de verdad del condicional, necesaria internamente en Matemática, no forma parte de las concepciones habituales.

Es frecuente que se asocie el condicional con la representación de relaciones causales. Cuando se quiere analizar si un fenómeno A es causa de otro B, hay que considerar, en cada caso de ocurrencia de A, si B también ocurre. Lo que importa en este análisis es que A ocurra; en términos lógicos, solo tiene interés el caso en el que el valor de verdad de la proposición "A ocurre" es cierto. En otras palabras, no es relevante preguntarse qué valor toma una proposición condicional cuando el antecedente es falso.

Esta es la clase de concepciones a las que Durand-Guerrier (2003) se vio enfrentada con sorpresa en su experiencia como docente en un curso de primer año universitario. Al proponer a sus estudiantes la determinación de los valores de una variable para los cuales una sentencia condicional resultaba cierta, encontró que solo unos pocos incluían en la respuesta los valores de la variable para los cuales el antecedente resultaba falso. Incluso pudo constatar que varios alumnos no se convencieron de que esta respuesta era correcta. En este sentido, es relevante la investigación de Alcock y Weber (2005) que destaca como uno de sus resultados que la aceptación de la validez de una argumentación descansa no solo en su estructura deductiva, sino además en la existencia de razones contextuales suficientemente fuertes para aceptar que las reglas de inferencia usadas son correctas. Esta constatación permite conjeturar plausiblemente que lo que hace sentido para el estudiantado, no toma por base un modo argumentativo aristotélico.

El ejemplo que sigue destaca la importancia que tiene la definición técnica del condicional en las demostraciones matemáticas; además, muestra cómo la posibilidad de construir y articular diferentes representaciones del mismo objeto matemático puede ayudar a superar las limitaciones que cada representación aislada tiene. P. N. Johnson-Laird (1984) al presentar la existencia de una controversia acerca de la forma en que los humanos razonan, plantea el estudio de las respuestas que se recogen al extraer conclusiones a partir de silogismos. Uno de ellos es el siguiente, en el que se pedía a estudiantes universitarios que extrajeran alguna conclusión, si había alguna, a partir de las sentencias:

a) Todos los apicultores son artistas.

b) Ningún químico es apicultor. 
Johnson-Laird afirma que "Algunos artistas no son químicos" es una conclusión a la que se arriba y destaca que ningún participante en el estudio la dio. Este resultado se enmarca en el llamado silogismo Darapti (Brunschvicg, 1945) y, como se verá a continuación, es incorrecto. En términos del cálculo de predicados, tomando $\mathrm{M}(\mathrm{x})$ como $\mathrm{x}$ es apicultor, $\mathrm{N}(\mathrm{x})$ como $\mathrm{x}$ es artista y $\mathrm{P}(\mathrm{x})$ como $\mathrm{x}$ es químico, podemos formular el silogismo anterior de la siguiente manera: $(\forall \mathrm{a})$ $(\mathrm{M}(\mathrm{a}) \rightarrow \mathrm{N}(\mathrm{a})) \wedge \neg(\exists \mathrm{a})(\mathrm{P}(\mathrm{a}) \wedge \mathrm{M}(\mathrm{a})) \rightarrow(\exists \mathrm{a})(\mathrm{N}(\mathrm{a}) \wedge \neg \mathrm{P}(\mathrm{a}))$. Sin embargo, esta conclusión es errónea de acuerdo con lo que se detalla a continuación:

I) Si se asume que no hay apicultores, (es decir M(a) toma el valor falso para cualquier a) entonces:

a) ( $\forall$ a) $(\mathrm{M}(\mathrm{a}) \rightarrow \mathrm{N}(\mathrm{a})$ ) es cierto, porque el condicional $\mathrm{M}(\mathrm{a}) \rightarrow \mathrm{N}(\mathrm{a})$ es cierto para cualquier a, porque su antecedente $\mathrm{M}(\mathrm{a})$ es falso para cualquier $\mathrm{a}$.

b) $\neg(\exists \mathrm{a})(\mathrm{P}(\mathrm{a}) \wedge \mathrm{M}(\mathrm{a}))$ es cierto, porque $(\exists \mathrm{a})(\mathrm{P}(\mathrm{a}) \wedge \mathrm{M}(\mathrm{a}))$ es falso, dado que la conjunción $\mathrm{P}(\mathrm{a}) \wedge \mathrm{M}(\mathrm{a})$ es falsa para cualquier a, porque $\mathrm{M}(\mathrm{a})$ es falso para cualquier a.

c) De acuerdo con i) y ii), $(\forall \mathrm{a})(\mathrm{M}(\mathrm{a}) \rightarrow \mathrm{N}(\mathrm{a})) \wedge \neg(\exists \mathrm{a})(\mathrm{P}(\mathrm{a}) \wedge \mathrm{M}(\mathrm{a}))$ resulta ser cierto.

II) Si se asume que no hay artistas (es decir, N(a) es falso para cualquier valor de a) o que todos son químicos (o sea, $\mathrm{P}$ (a) es cierto para cualquier a y entonces $\neg \mathrm{P}(\mathrm{a})$ es falso para cualquier a) resulta que la conjunción $\mathrm{N}(\mathrm{a}) \wedge \neg \mathrm{P}(\mathrm{a})$ es falsa para cualquier a, con lo que $(\exists a)(\mathrm{N}(\mathrm{a}) \wedge \neg \mathrm{P}(\mathrm{a}))$ es falso.

III) Con esto, el condicional resulta falso. ${ }^{8}$

Es interesante notar que si se acepta que existen apicultores entonces la fórmula $(\forall \mathrm{a})(\mathrm{M}(\mathrm{a}) \rightarrow \mathrm{N}(\mathrm{a})) \wedge \neg(\exists \mathrm{a})(\mathrm{P}(\mathrm{a}) \wedge \mathrm{M}(\mathrm{a})) \rightarrow(\exists \mathrm{a})(\mathrm{N}(\mathrm{a}) \wedge \neg \mathrm{P}(\mathrm{a}))$ resulta cierta, porque no es posible conseguir que el antecedente sea cierto y el consecuente falso.

En efecto, admitido que M(a) es cierto para algún a, digamos ao, para que el antecedente sea cierto debe ocurrir que $\mathrm{N}(\mathrm{a})$ tome el valor cierto para $\mathrm{a}_{0}$ (en caso contrario $(\forall \mathrm{a})(\mathrm{M}(\mathrm{a}) \rightarrow \mathrm{N}(\mathrm{a})$ ) resultaría falso). Con este resultado, si

\footnotetext{
${ }^{8}$ Existen otras asignaciones para conseguir que esta fórmula sea falsa.
} 
fuera el consecuente falso, $\mathrm{P}(\mathrm{a})$ debería ser cierto para ao (de lo contrario ( $\exists$ a) $(\mathrm{N}(\mathrm{a}) \wedge \neg \mathrm{P}(\mathrm{a}))$ sería cierto). Entonces, la fórmula $\neg(\exists \mathrm{a})(\mathrm{P}(\mathrm{a}) \wedge \mathrm{M}(\mathrm{a}))$ es falsa, dado que $\mathrm{P}(\mathrm{a}) \wedge \mathrm{M}(\mathrm{a})$ queda cierta para ao.

Es decir, lo que parece llevar al error que comete Johnson-Laird (1984) es la no consideración de un caso en el que una sentencia condicional es cierta porque su antecedente es falso. Sin embargo, aún en Matemática, las nociones de implicación y de prueba están principalmente asociadas a la consideración de los casos en los que las premisas, cuya conjunción constituye el antecedente, son ciertas. Tal como señalan Hoyles y Küchemann (2002), es necesario realizar una distinción entre la implicación material asociada con el conectivo lógico condicional y, las proposiciones hipotéticas, en las que se analiza si una sentencia (la conclusión) es consecuencia lógica de otras (las premisas) a partir de considerar cuál es el valor de verdad de la conclusión cuando todas las premisas son ciertas. Aun cuando ambas nociones están indisolublemente ligadas (una proposición hipotética se formaliza como una implicación material) esta asociación no es clara para los aprendices, e incluso para los profesores (Durand-Guerrier, 2003). Hasta aquí se ha manejado esta situación en el SMS propio del cálculo de predicados. Cerrando esta sección, se ejemplifican los procesos de conversión presentando, a continuación, un análisis del mismo silogismo, pero en el SMS de la teoría intuitiva de conjuntos.

Para esto se toma el universo $\mathrm{U}$ como el conjunto de todas las personas, $\mathrm{P}$ como el de los químicos, $\mathrm{N}$ como el de los artistas, $\mathrm{M}$ como el de los apicultores.

La premisa "Todos los apicultores son artistas" queda representada por el hecho de que $\mathrm{M} \subseteq \mathrm{N}$. La premisa "Ningún químico es apicultor" queda representada por el hecho de que $\mathrm{P} \cap \mathrm{M}=\emptyset$. La pretendida conclusión "Algunos artistas no son químicos" podría representarse como $\mathrm{N}-\mathrm{P} \neq \varnothing$ o, equivalentemente, como $\mathrm{N} \not \subset \mathrm{P}$. 


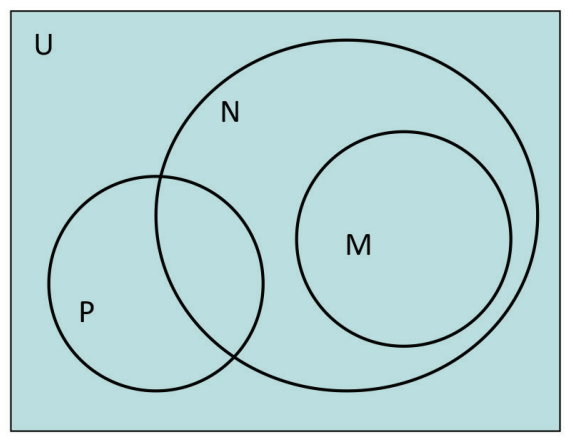

Diagrama 1

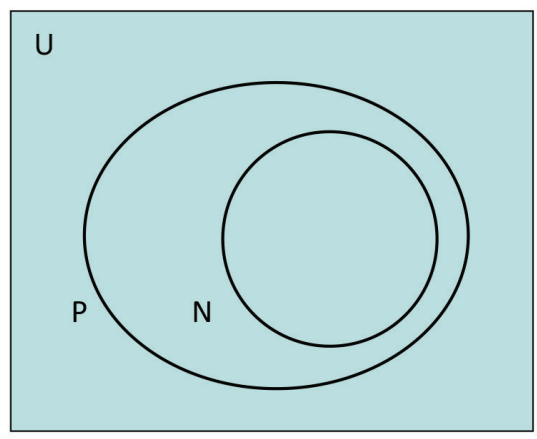

Diagrama 2

Así que, si $\mathrm{M} \neq \varnothing$ (es decir, "existen apicultores" es cierto), como $\mathrm{M} \subseteq \mathrm{N}$ y $\mathrm{P} \cap \mathrm{M}$ $=\emptyset$, entonces no puede ocurrir que $\mathrm{N} \subset \mathrm{P}$ (o sea, hay artistas que no son químicos), como se ve en el diagrama 1 . Ahora consideremos el caso que $\mathbf{M}=\varnothing$. Esto se representa en el diagrama 2 con la ausencia de referencias a $\mathbf{M}$ (este es un ejemplo de cómo cada registro de representación tiene limitaciones para dar cuenta de ciertos aspectos de lo representado: los diagramas de Venn no permiten, por su propia conformación, representar al conjunto vacío). Nada impide que $\mathrm{N} \subset \mathrm{P}$, lo que equivale a decir que "Todos los artistas son químicos" lo cual contradice la pretendida conclusión. Esto cierra el desarrollo del ejemplo, en el que se ha expuesto que cada representación presenta limitaciones y cómo la articulación de diferentes representaciones del mismo objeto contribuye a la comprensión del tema en discusión. Los ejemplos anteriores ilustran la coexistencia de diversos discursos en el aula y ponen de relieve que la Lógica Matemática emerge como disciplina (a fines del siglo XIX) para responder a problemas internos a la Matemática (teoría de conjuntos, formalización del análisis, entre otros). Algunas de sus construcciones, como la definición del condicional, son concesiones obligadas por la necesidad de coherencia interna. Sin embargo, otras necesidades o diferentes entornos pueden dar lugar a otras maneras de argumentar, no sólo en ámbitos externos a las Matemáticas, sino incluso internamente. Es en este sentido que algunos autores (Crespo, Farfán y Lezama, 2010) destacan que la presencia de algunas formas argumentativas en el aula no puede explicarse a partir de la lógica aristotélica, que sin embargo es la que subyace a las Matemáticas. 


\section{MOTIVACIONES PARA ESTA EXPERIENCIA}

Además de los señalados, un elemento destacable que emerge del trabajo de Alcock y Simpson $(2004 ; 2005)$ es la constatación de la existencia de dos clases de aprendices, aquellos que priorizan en su trabajo los aspectos de la representación algebraica, con poco o ningún apoyo en gráficas de algún tipo, y los que apelan principalmente a representaciones gráficas para visualizar las relaciones relevantes. Cabe entonces preguntarse acerca de la relación entre la dificultad de una tarea matemática y el sistema de representación en el que ha sido planteada. Por otro lado, en el discurso matemático del aula aparecen constantemente argumentos deductivos donde el reconocimiento de la relación entre dos enunciados (cuál es suficiente, necesario o suficiente y necesario para el otro) es crucial para establecer la validez del razonamiento en cuestión.

Adicionalmente, en la organización curricular de la enseñanza media superior en Uruguay el tratamiento de los temas de Cálculo Diferencial tiene un sesgo hacia la adquisición de habilidades de cálculo, mientras que los cursos universitarios iniciales cambian el foco a aspectos conceptuales, donde las nociones de demostración y de rigor juegan un papel principal. Por estos motivos, se eligió indagar acerca del uso por parte de los estudiantes de las nociones de condición necesaria o condición suficiente en formulaciones condicionales, presentadas o bien en el registro algebraico o bien en el gráfico, en situaciones como las que deben abordar en tareas usuales que se refieren a la definición de límite de una función en un punto, en los cursos universitarios iniciales de Cálculo. Se optó por un diseño que minimizara los cambios en el desarrollo habitual del curso, dado que se pretendía recoger información que pudiera ser útil en el desarrollo de intervenciones didácticas que incorporaran la enseñanza de temas de Lógica en el marco de los contenidos de Cálculo. Más específicamente, se decidió responder a las siguientes cuestiones:

1) ¿Qué uso de los enunciados condicionales (condición suficiente, condición necesaria) muestran ingresantes a la universidad?

2) ¿Qué registro de representación, gráfico o algebraico, presenta mayor dificultad en tareas con enunciados condicionales?

El marco conceptual para responder a estas preguntas está constituido, por un lado, por los aspectos destacados en la relación entre los SMS y la actividad 
matemática, y, por otro, con las nociones de condicional proporcionadas por la Lógica. A continuación, se describe esta experiencia.

\section{DISEÑO DE LA EXPERIENCIA}

\section{PARTICIPANTES}

Los participantes en esta experiencia fueron cuarenta estudiantes de primer año de carreras de ingeniería de la Universidad Católica del Uruguay (UCU), que asistían al primer curso de Cálculo. Se eligió esta muestra teniendo en cuenta que el estudio de Cálculo puede considerarse el punto de partida para el desarrollo del pensamiento matemático avanzado.

\section{INSTRUMENTOS}

Para esta experiencia se prepararon dos cuestionarios de ocho ítems con cuatro alternativas de respuesta cada uno. Ambos cuestionarios fueron elaborados de manera que la mitad de las preguntas están formuladas como condición suficiente y la otra mitad en modo de condición necesaria.

Estos cuestionarios fueron aplicados en la tercera semana del curso (marzo de 2017), en dos clases consecutivas, de sesenta minutos de duración cada una, antes del comienzo del tratamiento del tema de límite de una función en un punto. Con esta separación temporal se buscó evitar que se favoreciera la posibilidad de que los estudiantes los vincularan y por eso, se vieran eventualmente inducidos a llevar a cabo operaciones de conversión. Se indicó a los estudiantes que la relevancia de la tarea consistía en su relación con tema a seguir, el de límite, dado que les anticipaba algunas de las cuestiones que deberían resolver. Las respuestas de los estudiantes fueron registradas en hojas individuales que les fueron provistas, en las que debían marcar la opción que consideraban correcta.

La fiabilidad del cuestionario se estudió con un Alfa de Cronbach. La consistencia interna de los instrumentos fue aceptable, teniendo en cuenta que se trataba de pruebas de 16 preguntas (alfa de Cronbach de.59). Este análisis muestra que todos los ítems se comportan de manera similar, la supresión de alguno no aumenta la fiabilidad. 
En el Ilamado Cuestionario Gráfico (CG) las preguntas se referían a una función presentada a través de su gráfica cartesiana, de la que no se daba la fórmula algebraica. En el Ilamado Cuestionario Algebraico (CA) los ítems interrogaban acerca de una función presentada a través de su ecuación algebraica de la que no se daba la gráfica.

Los enunciados que se referían a cada condición usaban formatos diferentes:

a) para la condición suficiente se usaron "para que ocurra Q es suficiente que P", "P alcanza para Q", "si P entonces Q", "cuando ocurre P resulta Q".

b) para la condición necesaria se usaron "para que sea $\mathrm{P}$ debe ser $\mathrm{Q}$ ", "Q es necesaria para $\mathrm{P}$ ", "sólo si Q resulta P", "para estar seguro de que sea $\mathrm{Q}$, debe ser $\mathrm{P}^{\prime \prime}$.

Con el fin de garantizar que las preguntas estaban formuladas siguiendo las condiciones propuestas, dos profesores universitarios de Matemática clasificaron cada ítem según fuese de condición necesaria o suficiente.

Las alternativas de respuestas incorrectas fueron elegidas a partir de observaciones informales de situaciones de clase o de registros escritos de estudiantes en pruebas de evaluación en cursos anteriores de la misma asignatura, en las que, a partir del uso del lenguaje oral de los estudiantes, se detectaron formas de interpretar la estructura condicional que no eran las correctas. Otras de las opciones incorrectas de respuesta incluían desconocimiento de los contenidos matemáticos.

Se presentan algunos ítems de estos cuestionarios, con sus respectivos análisis didácticos.

Las preguntas del formulario algebraico se refieren a la función $f$ dada por cualquiera de las fórmulas siguientes $\mathrm{f}(\mathrm{x})=\mathrm{x}^{3}-3 \mathrm{x}+2$ o $\mathrm{f}(\mathrm{x})=(\mathrm{x}+2)(\mathrm{x}-1)^{2}$. Se incluyeron las dos fórmulas para simplificar el trabajo algebraico necesario para responder alguna de las preguntas.

Las dos preguntas que se presentan a continuación resultaron difíciles, registrando cada una un porcentaje del $45 \%$ de respuesta correctas.

La pregunta 7 es la siguiente:

La afirmación "Para que $f(x)$ sea positivo es suficiente que $x$ sea positivo y distinto de 1":

a) es falsa porque f(-1) es positivo y -1 no es positivo

b) es falsa porque $\mathrm{f}(\mathrm{x})$ es positivo para $\mathrm{x}$ mayor que $-2 \mathrm{y}$ distinto de 1 
c) es cierta porque, por ejemplo, 2 es positivo y $\mathrm{f}(2)$ es positivo

d) es cierta porque si $x$ es positivo y distinto de 1 resulta $f(x)>0$

En esta pregunta es explícita la condición de suficiente que se da a un enunciado ( $x$ es positivo y distinto de 1 ) respecto al otro (para que $\mathrm{f}(\mathrm{x})$ sea positivo). Sin embargo, el orden en el que se presentan estos enunciados no es el más frecuentemente utilizado. La respuesta correcta es la d). Quienes contesten las opciones a) o b), posiblemente estén confundiendo la condición considerándola necesaria (verosímilmente, debido al orden de los enunciados) Los que elijan la opción c) cometen el error lógico de pretender establecer la validez de un enunciado a partir de la verificación de casos particulares.

La pregunta 8 va a continuación:

Para estar seguro de que x está entre 0 al menos y 2 a lo sumo:

a) $f(x)$ debe estar entre 0 y 4 , ya que si $f(x)$ menor que 0 implica $x$ menor que -2 y $f(x)$ mayor que 4 implica $x$ mayor que 2 .

b) $f(x)$ debe estar entre 0 y 1 , ya que $f(0)=2$ y $f(1)=0$, ya que $f$ es monótona decreciente entre 0 y 1 .

c) $f(x)$ debe ser menor que 4 porque $f(x)$ es mayor que 4 si $x$ es mayor que 2 .

d) $f(x)$ debe estar entre 0 y 4 , ya que $f(0)=2$ y $f(2)=4$.

A diferencia de la anterior, la condición necesaria a la que refiere esta pregunta está implícita en el verbo "deber" utilizado. La respuesta correcta es la opción a). Quienes contestan la opción b) podrían estar considerando la condición como suficiente. En caso de marcar la opción c) posiblemente se esté cometiendo un error lógico al negar una conjunción. Finalmente, asumir la monotonía de la función en el intervalo entre 0 y 2 (lo que es erróneo) puede explicar la elección d).

Las preguntas del cuestionario gráfico se refieren a la siguiente gráfica. 


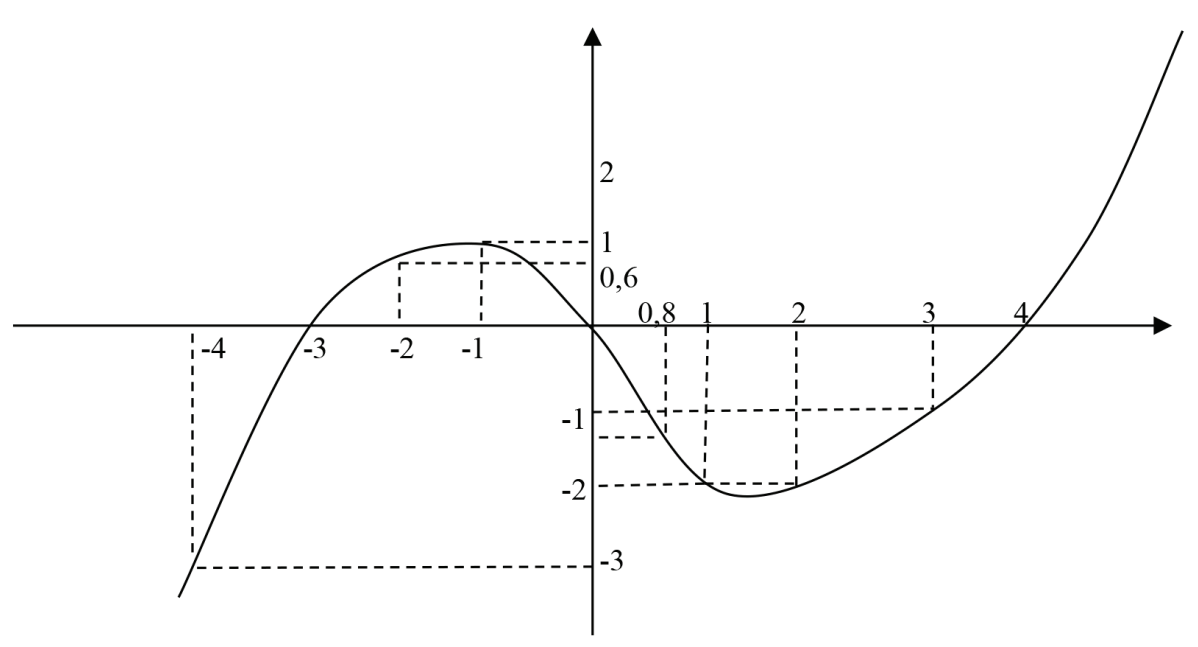

La pregunta 1 se da a continuación:

Es suficiente que $x$ esté entre -2 y 3 para que $f(x)$ esté:
a) entre -1 y 1 .
b) entre -3 y 2.
c) entre -2 y 1.
d) entre -1 y 0,6 .

Como en la pregunta 7 del cuestionario algebraico, se explicita el tipo de condición en el enunciado que se analiza. La diferencia entre estas dos preguntas está dada por la elección de los distractores. En las tres opciones incorrectas (a, c y d) los errores que explican que puedan ser elegidas están relacionadas con el contenido matemático involucrado (asumir monotonía de la función o hallar equivocadamente un valor).

La pregunta 5 es la siguiente:

Para que $\mathrm{f}(\mathrm{x})$ esté entre 0 y 1 , $\mathrm{x}$ debe estar

a) entre -3 y 0 o entre 4 y un número $\alpha$ (que es mayor que 4 y no se puede determinar a partir de la información de la gráfica) donde la función toma el valor 1. 
b) entre -3 y -1 .

c) entre -1 y 0 .

d) entre 0 y 1 .

La estructura de esta pregunta es similar a la 8 del cuestionario algebraico. En efecto, la condición de necesaria se expresa a través del uso del verbo deber; además, las opciones b) y c) podrían ser contestadas por quienes confundan la condición con suficiente, mientras que la d) sería elegida por quienes confundan los roles de las variables independiente y dependiente. La opción correcta es la a).

\section{ANÁLISIS DE LOS RESULTADOS}

En esta experiencia se consideraron dos variables independientes y una variable dependiente.

La primera variable independiente se refiere a los SMS y los valores que toma son registro gráfico y registro algebraico, dado que cada una de las preguntas del cuestionario está formulada en uno de estos registros. La segunda variable independiente es la estructura lógica y toma los valores condición necesaria y condición suficiente, dado que, al igual que con la variable anterior, cada pregunta del cuestionario está redactada de manera que plantea una de estas dos condiciones. El rendimiento es la variable dependiente, medida a partir del número de respuestas correctas en cada cuestionario. Se tiene entonces un diseño factorial intrasujeto $2 \times 2$, con una variable dependiente, que es el promedio del número de respuestas correctas. La tabla 1 muestra los resultados registrados en el rendimiento, en relación con las variables independientes. En cada casilla se indica el número máximo de respuestas correctas (4 u 8 según el caso).

Tabla 1. Rendimiento en relación con tipo de registro y tipo de condición

\begin{tabular}{llll}
\hline & Registro gráfico & Registro algebraico & Total por condición \\
\hline Condición necesaria & $3,2(4)$ & $2,57(4)$ & $5,77(8)$ \\
Condición suficiente & $2,28(4)$ & $2,03(4)$ & $4,31(8)$ \\
Total por registro & $5,48(8)$ & $4,6(8)$ & \\
\hline
\end{tabular}


Se analizaron estos datos con diferentes tipos de contrastes estadísticos, para indagar acerca de eventuales diferencias en el rendimiento en relación con las variables independientes.

Uno de ellos fue un ANOVA de medidas repetidas intrasujeto (SMS y estructura lógica, son los mismos estudiantes los que responden a todas las cuestiones) y sus interacciones. Resultó que el rendimiento en la combinación condición necesaria-registro gráfico es significativamente mayor que en cualquiera de las otras tres combinaciones (F15.1 nivel de confianza de 99\%). Las diferencias entre las otras combinaciones no resultaron significativas.

Otro contraste estadístico fue el uso del test t de Student para muestras relacionadas, que mostró, en ambos casos, que las diferencias son significativas (nivel de confianza de 99\%), a favor, respectivamente, del registro gráfico y de la condición necesaria.

En efecto, los estudiantes respondieron correctamente $58 \%$ de las preguntas sobre condición necesaria, mientras que el porcentaje de respuestas correctas sobre condición suficiente fue del $43 \%$. Similarmente, se constató un porcentaje de $55 \%$ de respuestas correctas en el registro gráfico, y en el algebraico este porcentaje fue del $46 \%$.

Estos mismos datos fueron analizados utilizando una prueba de los rangos con signos de Wilcoxon, y se obtuvieron los mismos resultados, es decir, existen diferencias significativas entre los rendimientos de los estudiantes cuando:

1) la tarea se presenta en un registro gráfico: resulta más fácil reconocer la estructura de condición necesaria que la de condición suficiente;

2) se compara globalmente el uso de las estructuras de condición necesaria y condición suficiente, resulta más fácil la primera que la segunda;

3) se compara globalmente el rendimiento, en el registro gráfico resulta mayor que en el algebraico.

Corresponde observar que los distractores elaborados para detectar errores sobre contenidos matemáticos tuvieron una muy baja frecuencia, lo que permite asociar los errores detectados con el uso del condicional. 


\section{CONCLUSIONES}

Los resultados del estudio muestran diferencias en el uso que los estudiantes hacen de los enunciados condicionales (condición suficiente, condición necesaria) y su relación con los Sistemas Matemáticos de Símbolos en los que se presenta la información sobre estos enunciados (registro gráfico, registro algebraico) en un curso de Cálculo de primer año en la universidad. Las tareas sobre condición necesaria presentadas en el registro gráfico resultaron significativamente más sencillas que las demás, se obtuvieron indicios de un mejor rendimiento en tareas presentadas en el registro gráfico respecto de las correspondientes al algebraico y resultaron mejores los desempeños en las tareas sobre condición necesaria en relación con las tareas sobre condición suficiente.

En relación con la primera cuestión planteada en este estudio, el uso de las sentencias condicionales que hacen estudiantes ingresantes a la universidad, se constató que el grado de dificultad registrado en las tareas propuestas es alto. El promedio de respuestas correctas en general no superó el $60 \%$. Ello alerta respecto de que gran parte de las expresiones del discurso del aula no estarían siendo comprendidas por los estudiantes; entre otras consecuencias, los estudiantes no están comprendiendo lo que se espera que entiendan a partir del discurso docente. Ello concurre con lo advertido por Camacho, Sánchez y Zubieta (2014) quienes han señalado que en los argumentos (incluso en los correctos) que utilizan estudiantes universitarios aparecen elementos contextuales o intuitivos y, difícilmente, se encuentra que ellos manejen las estructuras lógicas subyacentes.

Este es un hecho reseñado en la investigación en Matemática Educativa, y tiene explicaciones bastante diversas. Una de ellas radica en el carácter técnico de la lógica aristotélica en la que, en particular, la definición del valor de verdad del condicional responde a cuestiones que hasta cierto punto están alejadas de la experiencia cotidiana (Crespo, Farfán y Lezama, 2010). Otro argumento a considerar es el discurso en el aula, en especial, la forma en que son usados ciertos verbos que se asocian a la condición suficiente (alcanzar, bastar) o la necesaria (deber, tener que). Es posible que estos verbos sean usados en forma inadvertida, tanto por estudiantes como por profesores, indistintamente para cualquiera de las dos condiciones. También, en relación con el discurso, al menos en algunos idiomas como el español o el francés, puede señalarse que, en una sentencia condicional, los verbos deben conjugarse en diferentes modos, en el subjuntivo cuando el verbo aparece en el antecedente y en el potencial cuando está en el 
consecuente: "Si (verbo en modo subjuntivo) entonces (verbo en modo poten(ial)". Esta distinción de modos de conjugación resulta entonces asociada con el reconocimiento de dos partes asimétricas en la sentencia, de manera que una de ellas (el antecedente) es condición suficiente para la otra (el consecuente) o, equivalentemente, esta otra (el consecuente) es condición necesaria para aqueIla (el antecedente). Esta cuestión amerita una investigación específica, para establecer hasta qué punto la ocurrencia de ciertos verbos o de ciertas estructuras gramaticales puede incidir en el uso adecuado de las estructuras lógicas.

Los saberes de la vida diaria, los del currículo y los eruditos, establecen sus raíces en matrices de sentido de epistemes propias. Las metáforas de expresiones de condición y condicional en lo cotidiano aluden a estar sujeto, supeditado, subordinado, a ser dependiente. Por otra parte, esas palabras en el cálculo proposicional aluden a posibilidad, pudiendo haber otras opciones, otros caminos que se relacionan con el consecuente. Favorecer aprendizajes requerirá poner en diálogo estas distintas coherencias, articulándolas, con base en diseños científicos de enseñanza. En cualquier caso, resulta que, si se pretende que los aprendices utilicen elementos de Lógica Matemática en sus actividades y que, en particular, recurran al uso del condicional, deben concederse espacios para la enseñanza intencional de estos temas.

En ese sentido van propuestas como la de Durand-Guerrier (2005) para utilizar situaciones habituales de los cursos de Matemática de manera de enfatizar acerca del uso de sentencias condicionales y cuantificadores en los procesos de demostración, explicitando las reglas de inferencia usadas, o la de Morou y Kalospyros (s.f), diseñando un curso de lógica orientado a estudiantes en la enseñanza media en el que, entre otros focos, se enfatizó la traducción formal de argumentaciones en las que aparecen sentencias condicionales tomadas de diversas fuentes, incluyendo desde publicaciones recreativas hasta actividades en un curso de Cálculo.

En relación con la segunda cuestión estudiada, las diferencias detectadas que refieren al registro de representación son también de importancia didáctica. Es destacable que los resultados de este estudio muestran que el uso del registro gráfico y el reconocimiento de la condición necesaria resultan más sencillos que el uso del registro algebraico y el reconocimiento de la condición suficiente, respectivamente, y que la combinación condición necesaria-registro gráfico es la que con gran diferencia resulta la más fácil. La enseñanza tradicional ha privilegiado el uso del registro algebraico en detrimento de otros registros de representación. Por ejemplo, a una demostración basada en un diagrama de Venn 
correspondiente a un registro gráfico, no se le reconoce el mismo estatus de rigor matemático que a otra desarrollada en el registro algebraico. Teniendo en cuenta los resultados de este estudio, resulta una oportunidad revisar las prácticas de enseñanza buscando un balance más equilibrado en el uso de los registros de representación para favorecer el aprendizaje. Una cuestión a abordar, por lo tanto, es la de construir y poner a prueba diseños instruccionales que insistan en la articulación de diferentes registros, al menos, el gráfico y el algebraico, como los que proponen Blázquez y sus colaboradores (Blázquez, Ortega, Gatica y Benegas, 2006).

De modo consistente aparece el registro gráfico asociado con un mejor desempeño en las tareas. Parafraseando a Radford y Puig (2007, p. 146) "el significado de los cálculos era asegurado por transformaciones geométricas visuales", ${ }^{9}$ una posible explicación de esta diferencia es la forma en la que la gráfica de una función permite el acceso a la información, comparada con la de una ecuación. Por ejemplo, la decisión acerca de si la imagen de un cierto subconjunto del dominio está contenida en un cierto conjunto del recorrido puede tomarse a partir de verificar que los puntos de la gráfica se encuentran en un cierto rectángulo. Incluso algunas de estas operaciones pueden verse favorecidas por la posibilidad de ejecutar gestos, como señalar partes del dominio o del codominio, que simplifican el proceso de establecer relaciones entre estos subconjuntos.

En las formulaciones algebraicas se requieren tratamientos (en el sentido de Duval, 1998), a veces engorrosos y prolongados, antes de poder explicitar las relaciones entre las variables; entre otras situaciones, cuando el resultado de una inecuación es la unión de dos intervalos, puede ser difícil reconocer sus subconjuntos, lo que es relevante para decidir, entre dos enunciados dados, si uno de ellos es condición es suficiente o necesaria para el otro. Esto podría contribuir a explicar que las diferencias entre condición necesaria y condición suficiente se vean opacadas en este registro. No slo resultan más difíciles las tareas en el registro algebraico que en el gráfico, sino que, además, no se encuentra en el registro algebraico el reconocimiento de la distinción entre condición necesaria y condición suficiente, distinción que se reconoce en el registro gráfico. Una de las cuestiones que este estudio deja abierta es el grado en que una aproximación a la enseñanza con un énfasis mayor en representaciones gráficas podría favorecer los aprendizajes. Al menos algunas nociones

\footnotetext{
9 Traducción de los autores
} 
relacionadas con el concepto de función y las sentencias condicionales parecen resultar más accesibles desde este registro. Desde el punto de vista didáctico, un desafío es estudiar las relaciones entre el condicional y los SMS en términos de otros contenidos matemáticos. La definición de independencia lineal de vectores, en la que explícitamente resulta necesario estudiar una sentencia condicional, puede presentarse tanto algebraica como geométricamente ya sea en el plano o en el espacio; otro ejemplo se presenta en torno a la definición de monotonía de funciones reales con dominio real.

\section{REFERENCIAS}

Alcock, L., y Weber, K. (2005). Proof validation in real analysis: Inferring and checking warrants. The Journal of Mathematical Behavior, 24(2), 125-134.

Alcock, L., y Simpson, A. (2004). Convergence of sequences and series: Interactions between visual reasoning and the learner's beliefs about their own role. Educational Studies in Mathematics, 57(1), 1-32.

Alcock, L., y Simpson, A. (2005). Convergence of sequences and series 2: Interactions between nonvisual reasoning and the learner's beliefs about their own role. Educational Studies in Mathematics, 58(1), 77-100.

Arcavi, A. (2003). The role of visual representations in the learning of mathematics. Educational Studies in Mathematics, 52(3), 215-241.

Artigue, M. (1995). La enseñanza de los principios del cálculo: problemas epistemológios, cognitivos y didácticos. En M. Artigue, R. Douady, L. Moreno, y P. Gómez (Eds.), Ingeniería Didáctica en Educación Matemática (pp. 97-140). Grupo Editorial Iberoamérica.

Artigue, M. (2000). Teaching and learning calculus. What can be learned from education research and curricular changes in France. En E. Dubinsky, A. Schoenfeld, y J. Kaput (Eds.), Research in Collegiate Mathematics Education IV (pp. 1-14). CBMS.

Berger, M. (2004). The funtional use of a mathematical sign. Educational Studies in Mathematics, 55(1), 81-102.

Blázquez, S., Ortega, T., Gatica, S. N., y Benegas, J. (2006). Una conceptualización de límite para el aprendizaje inicial de análisis matemático de la universidad. Revista Latinoamericana de Investigación en Matemática Educativa, 9(2),189-209.

Brunschvicg, L. (1945). Las etapas de la filosofía matemática. Lautaro.

Camacho, V., Sánchez, J., y Zubieta, G. (2014). Los estudiantes de ciencias, ¿pueden reconocer los argumentos lógicos involucrados en una demostración? Enseñanza de las Ciencias, 32(1), 117-138. 
Camarena, P. (2010). Aportaciones de investigación al aprendizaje y enseñanza de la Matemática en ingeniería. En Ingeniería en Comunicaciones y Electrónica (pp. 1-47). Academia de Ingeniería de México. http://www.ai.org.mx/ai/archivos/ingresos/camarenagallardo/dra._patricia_camarena_gallardo.pdf

Crespo, C., Farfán, R. M., y Lezama, J. (2010). Argumentaciones y demostraciones: una visión de la influencia de los escenarios socioculturales. Revista Latinoamericana de Investigación en Matemática Educativa 13(3), 283-306.

Douady, R. (1984). Jeux de cadres et dialectique outil-objet dans l'enseignement mathématique. Une réalisation dans tout le cursus primaire (Tesis doctoral no publicada). Université de Paris VII.

Douady, R. (1986). Jeux de cadres et dialectique outil-objet. Recherches en Didactique des Mathématiques, 7(2), 5-32.

Durand-Guerrier, V. (2003). Which notion of implication is the right one? From logical considerations to a didactic perspective. Educational Studies in Mathematics, 53(1), 5-34.

Durand-Guerrier, V. (2005). Natural deduction in predicate calculus a tool for analysing proof in a didactic perspective. En M. Bosch (Ed.), Proceedings of the Fourth Congress of the European Society for Research in Mathematics Education (pp. 409-419). ERME.

Duval, R. (1995). Sémiosis et pensée humaine. Peter Lang.

Duval, R. (1998). Registros de representación semiótica y funcionamiento cognitivo del pensamiento. En F. Hitt (Ed.), Investigaciones en Matemática Educativa II (pp. 173201). Grupo Editorial Iberoamérica.

Gagatsis, A., y Shiakalli, M. (2004). Ability to translate from one representation of the concept of function to another and mathematical problem solving. Educational Psychology, 24(5), 645-657.

Glymour, C. (1998). Thinking things through: An introduction to philosophical issues and achievements. The MIT Press.

Hoyles, C., y Küchemann, D. (2002). Students' understanding of logical implication. Educational Studies in Mathematics, 51(3), 193-223.

Inglis, M., y Alcock, L. (2012). Expert and novice approaches to reading mathematical proofs. Journal for Research in Mathematics Education, 43(4), 358-390.

Johnson-Laird, P. N. (1984). El pensamiento como habilidad. En M. Carretero y J. Madruga (Eds.), Lecturas en psicología del pensamiento (pp. 123-145). Alianza Psicología. Kaput, J. (1987). Towards a theory of symbol use in mathematics. En C. Janvier (Ed.), Problems of representations in the teaching and learning of mathematics (pp. 159196). Laurence Earlbaum Associates.

Kieran, C. (1992). The learning and teaching of school algebra. En D. A. Grouws (Ed.), Handbook of research on mathematics teaching and learning (pp. 707-762). Macmillan. 
Lew, K., Fukawa-Conelly, T. P., Mejía-Ramos, J. P., y Weber, K. (2016). Lectures in advanced mathematics: Why students might not understand what the mathematics professor is trying to convey. Journal for Research in Mathematics Education, 47(2), 162-198.

Martí, E., y Pozo, J. I. (2000). Más allá de las representaciones mentales, la adquisición de sistemas externos de representación. Infancia y aprendizaje, 23(90), 11-30.

Morou, A. P., y Kalospyros, N. A. E. (s.f). The role of logic in teaching, learning and analyzing proof. Recuperado en enero 13, 2021 de http://www.cerme7.univ.rzeszow. pl/WG/1/CERME7_WG1_Morou\&Kalospyros.pdf

Palmer, S. E. (1977). Fundamental aspects of cognitive representations. En E. Rosch y B. Lloyd (Eds.), Cognition and categorization (pp. 259-303). Laurence Earlbaum Associates.

Pocoví, M. C., y Collivadino, C. (2014). Traducción entre lenguajes simbólicos de distintas áreas del conocimiento: el caso del flujo del campo eléctrico. Enseñanza de las Ciencias, 32(2), 53-69.

Puig, L. (2003). Signos, textos y sistemas matemáticos de signos. En E. Filloy (Ed.), Matemática educativa: aspectos de la investigación actual (pp. 174-186). Fondo de Cultura Económica.

Radford, L. (1998). On signs and representations. A cultural account. Scientia Paedagogica Experimentalis, 35(1), 277-302.

Radford, L., y Puig, L. (2007). Syntax and meanings as sensuous, visual, historical forms of algebraic thinking. Educational Studies in Mathematics, 66(2), 145-164.

Rojas, P. J. (2015). Objetos matemáticos, representaciones semióticas y sentidos. Enseñanza de las Ciencias, 33(1), 151-165.

Selling, S. K. (2015). Learning to represent, representing to learn. The Journal of Mathematical Behavior, 41, 191-209.

Tall, D. (1990). Inconsistencies in the learning of calculus and analysis. Focus on Learning Problems in Mathematics, 12(3-4), 49-63.

Weber, K., y Alcock, L. (2004). Semantic and syntactic proof productions. Educational Studies in Mathematics, 56(2), 209-234.

Zazkis, D., Weber, K., y Mejía-Ramos, J. P. (2015). Two proving strategies of highly successful mathematics majors. The Journal of Mathematical Behavior, 39, 11-27.

\section{EDUARDO MARIO LACUES APUD}

Dirección Postal: Maipú 2026, Montevideo, CP 11600 